\title{
Regulation and social practice online
}

Jenny Kennedy ${ }^{\mathrm{a}}$, James Meese ${ }^{\mathrm{b}}$, and Emily van der $\mathrm{Nagel}^{\mathrm{c}}$

${ }^{a}$ University of Melbourne, ${ }^{b}$ University of Technology, Sydney; ${ }^{c}$ Swinburne University of Technology

This article argues that everyday media practices are foundational to regulation on social media platforms. Beginning from a practice theory perspective, supported by qualitative research conducted on Facebook and Reddit, this paper shows how individual interactions with the platform and with other people on the site shape central regulatory norms on these sites. We suggest that our focus on practice complements existing studies that consider how regulation operates on social media platforms (see Gillespie, 2014) and shows how both practices and algorithms operate in conversation with one another in order to govern these sites. This research sets out an alternative trajectory of regulation, which is not based in law or privately established processes (such as EULAs, ToS or 'flags') but instead one grounded in the everyday practices of sociality, reciprocity, and perhaps even the maintenance of a particular community ideal.

Keywords: Facebook, Reddit, practice theory, media regulation, legal cultures, norms.

\section{Introduction}

This article applies practice theory to social media regulation. Existing studies of regulation on social media platforms tend to emphasise formal computational and legal structures, including algorithms, which are encoded procedures for transforming data into a desired 
output, based on specified calculations (Gillespie 2014); platform policies; and broader regulatory frameworks (Gillespie and Crawford 2014). This literature sets out a useful landscape of regulation, but it lacks an understanding of how individuals engage with, and negotiate, this terrain. In this paper, we carefully examine the interactions between the everyday practices of social media users and its formal structures, and, in doing so, offer an alternative perspective on how power is both constituted and deployed online. We suggest that practice plays a central role in shaping regulation online (following Murray et al. 2014), and that people on social media platforms tend to defer to established forms of practice rather than more formal methods of regulation (see Meese 2014), a claim supported by two detailed case studies.

This article directly responds to Nick Couldry's (2012) call for more media studies research that uses social practice theory, and builds on the recent work of legal scholars who have used practice theory to understand how law functions. Such scholars contend that 'local practices or norms are foundational and persistent', rather than 'ancestral or supplemental' (Murray et al. 2014, 6) to existing legal frameworks. We argue in this paper that this conceptual position is equally applicable to the development of regulatory frameworks online. We begin with a brief review of the literature, focussing on recent work that emphasises the formalising elements of practice and its role in shaping cultures of regulation online. Our argument is then developed through an exploration of specific online practices on the social media platforms Facebook and Reddit. In our conclusion, we outline how practices help to shape specific performances on such sites, and cumulatively assist in normalising particular types of desired performance.

\section{Background}


Practice theory is an attempt to understand the world by attending to the dynamic aspects of social life. We define practice in relation to the contemporary research that develops practice theory (see Shove and Pantzar 2005); work which aims to describe social orders and social constitution of individuals. This theoretical framework treats 'routines, shared habits, technique and competence' (Shove and Pantzar 2005) as central objects of analysis, and contends that these elements have a bearing on the constitution of natural and social worlds, rather than this being determined by institutions and other abstract structures (see Bourdieu 1984). This stance directly counterposes abstract concepts such as 'social structure' or 'regulation', and instead positions these formal structures as dynamic infrastructures that are 'manufactured daily, haphazardly, and laboriously in individual interactions' (Murray et al. 2014).

This theoretical approach is spread across a loosely grouped body of literature, and indeed a common criticism of practice theory literature is that it 'fails to make clear just what social practices are' (Barnes 2001, 17-28). In other words, it is methodologically problematic to reduce practice into a distinct set of elements that can be methodically applied across diverse phenomena. However, scholars have managed to localise particular disciplinary understandings and conceptualisations of practice, and it is this sort of contextual knowledge which can be of use to further scholarly work. As our focus in this paper is online social media platforms, norms, and regulation, we consider practice by drawing on recent research in media studies (Couldry 2012) and law (Murray et al. 2014) that engages with practice theory in ways that pay attention to dynamics of power.

We first turn to the work of media scholar Nick Couldry (2012), who deploys social practice theory in order to better understand the everyday uses of digital media. Couldry 
notes that a turn towards practice usefully avoids both the media text and media institutions to instead focus on a basic question: 'what are people (individuals, groups, institutions) doing in relation to media in the contexts which they act?' (Couldry 2012, 34). This allows for a comprehensive account of media consumption and distribution, and avoids an excessive focus on media audiences or the 'indeterminacy' of activities located around a specific media text (for example, watching televised sport). Instead, Couldry suggests that by approaching media through a practice-oriented perspective, a range of new actions emerge - such as 'searching',' showing' or 'presencing' - that may have been missed by other methodological perspectives.

This media-oriented approach underlines the productive interactions that can occur between social practice theory and established political economy analyses. Practice theory is able to provide a targeted and nuanced account of media circulation, and assist in mapping complex and dynamic spaces (for example, social media platforms). However, practice theory also allows us to challenge these aforementioned institutional structures more directly. In their analysis of intellectual property law, Laura Murray, S. Tina Piper, and Kirsty Robinson (2014) show how practices do not just reproduce formal institutional structures, but also present a range of negotiations between actors that in some cases replicate, and in others deviate, from law. This distinction underlines the foundational nature of practice, positioning practice as a formalising structure in its own right, rather than supplementary to existing institutions.

We suggest that this insight is equally applicable to the role of regulation on social media platforms. As we have already briefly noted, the current literature in media studies research has considered these issues in some detail, examining how platforms present 
particular discourses of engagement to their respective audiences (Burgess 2014) and how algorithms and reporting tools assist in regulating these ostensibly 'social' spaces (Gillespie 2013; Gillespie and Crawford 2014). These approaches largely present useful macro accounts of how particular institutions, such as newspapers, the wider tech community, algorithms, or social media platforms impact or attempt to influence the agency of individuals (such as Facebook and Reddit users). More recent work has examined the phenomenon of 'platform vernacular' (Gibbs et al 2014), where specific practices become expected and established vernaculars on specific social media platforms.

This work identifies particular regulatory agencies, but it does not engage substantially with their implications. In short, how are these vernaculars enforced and maintained? Gillespie himself recognises that there is more work to be done on algorithms, for example, noting that scholars need to 'unpack the warm human and institutional choices that lie behind these cold mechanisms' (Gillespie 2013, 169). We therefore contribute to this body of scholarship by offering a series of accounts, which outline people's 'microinteractions' (Murray et al. 2014, 7) on these sites. We shift the lens to practice, and identify this as a foundational site of regulation in terms of establishing normative behaviour, wider social discourses, and formal regulatory texts. This approach allows the insights of practice theory to directly further our understanding of how social media platforms are regulated, by outlining how individuals negotiate these broader institutional attempts at encouraging particular behaviours.

\section{Methods}

The data discussed in this paper are drawn from two qualitative studies of social practices on social media platforms. The first study examines how social practices help to shape 
normative imaginaries of sharing on Facebook. Through qualitative analysis of semistructured interviews with Facebook users, conducted between 2011 and 2013 as part of a larger study on sharing practices, we detail how performances of sharing and reciprocity negotiate norms of sharing, and establish undesired performances as oversharing.

The sample for this study consisted of 22 participants, recruited through convenience and snowball sampling, and across cultural and demographic differences. The participants range in age from 18 to 56. All participants reside in Melbourne, Australia. Transcripts for the semi-structured interviews were coded thematically (Flick 1998) within a framework of inductive reasoning. Semi-structured interviews were the primary method employed in this study for their suitability in bringing new understanding to practices and the discourses around those practices (Flick 1998; Holstein and Gubrium 2002). In interviews, participants were asked what the term sharing meant to them; where, how, and with whom they share; how they think others may perceive them through their sharing practices; how roles and relationships mediate their sharing practices; and how interfaces such as Facebook mediate their sharing practices.

The second study also explores normalisation, in an examination of Gonewild, a subreddit on social media platform Reddit. Nude or semi-nude self-portrait photographs submitted to the subreddit by pseudonymous posters were collected over a year long period between August 2013 and August 2014. This involved taking screenshots of the six main algorithms each day, and recording the top ten posts to the site, which included the title, main image, number of upvotes, and comments. Using content analysis, the systematic categorising of units of a text (Krippendorf 2013; Silverman 2011), the images were coded by gender, which was self-reported through the tags [m] for male or [f] for female; whether their 
face was in the image or not; how clothed they were; and the main body part shown in the image. Critical discourse analysis, which 'focuses on the form and content of communicative artifacts and the social practices that inform them' (Milner 2013, 2362), was deployed to understand how the images were created, how they were submitted to the site, and how they were received by both the audience who voted on the image and the algorithm which assigned the image its total votes, affecting where it would appear on the subreddit.

Through content analysis and critical discourse analysis, we detail how the practices of Gonewild participants and algorithms help to co-produce normative bodily standards, further underlining how the dynamic space of practice can contribute to the shaping of particular normative goals.

\section{Socially learning sharing practices on Facebook}

As one of the most heavily used social media platforms in the world, Facebook has a significant interest in formalising and encouraging particular forms of behaviour (van Dijck 2013). This can be done overtly through the use of various tools, which imagine a particular context of engagement occurring on Facebook: flags provide mechanisms for reporting offensive content to a social media platform within the predetermined rubric of a platform's community guidelines (Crawford and Gillespie 2014), like buttons allow individuals to approve of particular content, and the share button encourages the distribution of content across social networks. Kate Crawford and Tarleton Gillespie (2014) suggest that the practice of 'flagging' content tends to leave power in the hands of social media platforms. They also rightly note that the 'flags' cannot make the nuanced decisions that everyday social life requires. However, as our following analysis of sharing outlines, social practice plays a key 
role in establishing contexts for participation and normative behaviour online, and charts a parallel practice-led trajectory of regulation of social media content and engagement.

People have clear conceptual ideas about how Facebook's platform interface is designed to initiate sharing (Kennedy 2013). For instance, the 'share' button allows individuals to post something they see on Facebook to their own profile. Interfaces of social media platforms structure sharing practices through their information architecture, which is the hierarchy of particular elements and pathways on screen, to identify and highlight particular actions afforded in the interface. Interface structures are distinct from but interwoven with social structures, which both have bearing on practices. For example, one participant Michelle, a 35-year-old personal trainer, is unconvinced by the purpose of the 'share' button on Facebook:

My friend was talking to me about the 'share' button on Facebook. He doesn't like that because he feels that the notion of sharing is a positive notion that you're giving something whereas he feels like it justifies people to be in a bad mood or look like a loser and put that up and share, so that's a good thing! Whereas, actually, it's not a good thing. It should say 'share positive things' (Michelle, in interview, 2011).

Michelle and her friend feel that labelling this feature 'share' implies that anything posted through this function is coded as being meaningful and significant, and that it encourages people to post greater amounts of content to their profiles, which may in actual fact be trivial, contradictory, or create 'noise' in their presentation of self. Michelle's and her friend's conversation express the interplay of social norms with technological infrastructure. 
The interface is itself a collaborator in impression management (van Dijck 2013). As another participant, Esel, explains:

If you like something it is automatically shared with all your friends to say 'this person liked this, you should like it as well' so they are definitely directing you in which ways to share stuff (in interview, 2012).

Esel questions the way in which her use of the 'like' button is shaped by the algorithms of the platform which codes her 'like' activity and displays it to her friends in a way that suggests because she likes a particular brand of clothing, they might too. Her main concern is that it shows no scale of how much she likes the brand or item. The consequence is being presented as having a strong association with the brand or item, signalling aspects of taste that is not necessarily representative of her values. Additionally, the use of 'like' buttons, and other such counters, are primarily tracking devices proving valuable user metrics, fuelling the 'like economy' (Gerlitz and Helmond 2013).

Understanding platform conventions for sharing are as important as understanding social conventions. Regrets around sharing may be related to the disclosure of sensitive content, actioned without sufficient consideration, in spite of consideration of the consequences, or through misinterpretation of the interface. Indeed, Yang Wang et al. observe that people's 'regrettable postings' could be averted 'if they were better able to utilize Facebook's granular access controls' (Wang et al 2011, 10). Limitations of understanding particular interfaces, coupled with emotional states, contribute to sharing regrets. Yet regrets are not always motivated by a lack of comprehension of appropriate conventions. For instance, Vanessa, a 29-year-old university lecturer, had a conversation on Facebook, which she later deleted to establish a more comfortable boundary of accessibility 
for herself. She had posted a status update about wanting to go rock climbing, and an old friend with whom she had little contact in recent years responded, which extended into a conversation back and forth across their profile pages:

We kept forgetting that we were just on comment threads and there would be threads that were sixty, seventy comments long. Completely inane stuff, so not stuff I was worried about anyone else reading [...] It didn't really bother me that it was all public, though I did delete the thread. I'm not really sure why to be honest, just the fact that there was this long thread on my Facebook (Vanessa, in interview, 2011).

Even though the exchange didn't contain particularly sensitive details, its very banality and everydayness meant it was too mundane to be special enough to share, but specific enough to feel personal. While the effect of leaving the exchange up on her page would likely be minimal, to retain it risked signalling an inability to understand the conventions of the platform, or a form of arrogance by parading the conversation, and relationship it implied. Vanessa has learnt the conventions of sharing practices on the platform through her observation and engagements with others.

Sharing practices are socially learned (see Merten 1999). Examining users new to Facebook, Moira Burke, Cameron Marlow, and Thomas Lento (2009) established that people modified their sharing practices based on the performances modelled by their peers. Furthermore, the performances modelled in the first two weeks of Facebook use shape future sharing practices of the new users (Burke, Marlow, and Lento 2009), highlighting processes of social enculturation for sharing norms. Surrounding sharing is a complex set of tacit rules related to what can be shared, and with whom it can be shared. What has been dubbed 'oversharing' is the transgression of boundaries of disclosure, which create feelings of 
unease. Sharing provokes anxiety about its reception, especially when something personal. This is implied in Michelle's remark: 'You don't want to do any oversharing on Facebook' (Michelle, in interview, 2011).

The phantom of sharing is the fear of 'oversharing'. While realisation of having overshared can provoke anxiety and discomfort, enduring another's oversharing can elicit similar responses. Oversharing locates people in an uncomfortable situation where they feel obliged to reciprocate, but would rather not because they feel a threshold has been unduly crossed. Reciprocation to performances of oversharing risk endorsing and normalising that sharing and so non-reciprocation of oversharing is intended to convey disapproval to the offending party. One participant, Jasper, an 18-year-old student, describes how he observes this occurring on Facebook:

If someone [posts a Facebook] status that was somehow perceived to be really day to day, mundane or not really interesting or not worth sharing on Facebook - whatever that means, anyone can post whatever they want supposedly - others make a comment like 'cool story bro' or they'd make some sort of smart-arsed sarcastic comment (Jasper, in interview, 2012).

Jasper witnesses group censorship of undesired sharing practices which function to shame and humiliate the offender, and serve as a warning to others. While the interface itself appears unbiased in terms of perceptions of interest, there are social conventions, which are strongly enforced through reciprocation tactics. Reciprocity is a form of social control, negotiating and reinforcing norms of sharing.

Drawing from this study of sharing practices on Facebook, we are able to outline how sharing practices establish and assert social norms on Facebook. Oversharing is one way in 
which established practices are valued by individuals over regulation: it is not against Facebook's terms of service agreement to volunteer detailed banalities about one's life, but it is socially inappropriate. When sharing on Facebook is framed by the 'share' button, or by the blank text box at the top of the page prompting a response to 'What's on your mind?', these are presented as open-ended actions to be performed by any user, any way they interpret the prompt. But in contemporary networked culture, being an effective digital subject requires negotiating both the platform affordances and its norms. The practice of reciprocity as a way to shame, and therefore deter, oversharing illustrates the importance of social practice in establishing contexts for participation. Turning next to a study on Reddit Gonewild, we are able to detail how the interplay between regulation and social practices produce norms that shape the content on the site.

\section{Creating norms through social practices on Reddit Gonewild}

Reddit is a social media site that promotes user-generated content. Reddit users, known as Redditors, can submit content to various subreddits, or sub-forums, which is then ranked by user votes and algorithms. Each Redditor can allocate posts either one upvote or one downvote: the premise is that popular content will rise to the top of the page, while less popular content will sink. This means that Reddit appears as an ever-changing list of links, text posts, and images, with the goal of giving its audience a real-time account of the most popular content from across the internet.

Reddit Gonewild is one in a cluster of several pornography-themed subreddits, dedicated to original photographs of nude or semi-nude bodies. The page appears as a list of titled links to images, with a thumbnail preview of each image on the left, next to a numerical score indicating the total amount of 'karma' points or upvotes each post has received. Similar 
to the prominence of Facebook's 'What's on your mind?' text box, the largest text on Reddit Gonewild appears as a green box on the right-hand side, with 'SUBMIT A NEW LINK' written on it in capitals. Both of these boxes encourage people to contribute to the site, and both appear at first glance to accept any kind of content that someone may choose to provide. But practices on these platforms occur within a framework of socially enforced norms, coproduced by existing practices and algorithms. The Gonewild homepage consistently features posts of nude or partially nude women, most of whom are white, young, and slender, a normative standard that has been established through a year of qualitative content analysis. Although men, and other genders, can post their own photographs, they may interpret the frequency of women to mean that their photos would not be upvoted, and decide not to post them at all, reinforcing the demographic of the homepage.

Content on each subreddit is also sorted by six different algorithms, the default presenting the top 25 posts listed under 'hot'. The others appear as tabs at the top of the page: 'new' displays content in order of submission time; 'rising' is for posts that are quickly gaining upvotes; 'controversial' posts have a similar number of upvotes and downvotes; 'top' lets people further sort the content by featuring the most popular posts from the current hour, day, week, month, year, or all time; and 'gilded' posts have been awarded Reddit Gold, a USD \$4 month-long subscription to special features of the site. Algorithms sort content according to mathematical formulas, but they also assert power over the content by selecting some images to become visible, which, as Taina Bucher (2012) argues, is framed as a reward. The algorithms on Reddit mean the content is unlikely to stay where it is originally posted: content in 'new' will either receive upvotes, being elevated through 'rising' to 'hot', or will be downvoted, and arrive in 'controversial'. Content moving through algorithms means it is 
constantly being renewed, and, most significantly, means that non-conforming images are quickly pushed aside.

Not all Redditors choose to view the content through algorithms other than the default, and some are unaware that Reddit even employs algorithms: programmer Todd Schneider (2014) was surprised to find that Reddit was hardly a 'meritocracy' of upvotes minus downvotes, but was subject to many other factors, including what time it was submitted, and to which subreddit. These algorithms are never neutral, but represent subjective choices made by those who own and run Reddit. Programmer Amir Salihefendic (2010) explains that the 'hot' algorithm works by heavily weighting the first votes: the first ten upvotes have the same weight as the next 100. It also takes the time of posting into consideration: each post has its age in seconds divided by 45,000 - the number of seconds in 12.5 hours. This makes older posts gradually sink even if they are receiving tens of thousands of upvotes, keeping the homepage fresh. The 'controversial' algorithm features posts with similar numbers of upvotes and downvotes, which may explain why more photographs of women wearing lingerie are highly upvoted, compared with more explicit photos of spread vulvas: the more bland the image, the harder it is to take offence. 'Controversial' is where to find images that depict bodies other than that of young, white, slender women: the bodies that do not fit the norms of the site. Don Slater, in an ethnography of trading naked photographs on Internet Relay Chat (IRC), argues that the photographs represented a variety of sexual desires, but they were constrained by policed normative ideas. Slater claims that body representation on IRC 'is about grounding ideal identities, not about undercutting gender conventions' (Slater 1998, 97). 
These 'ideal identities' can also be seen on Reddit Gonewild, being actively controlled by the subreddit's audience, who can interact with content on the site by voting or commenting on it; sharing it by sending a link to another Redditor; saving it to their own profile; hiding it; or reporting it to the moderators to be removed. Just as a sarcastic reply to an oversharing post on Facebook dismisses it and rebukes the original poster, content is policed on Reddit through downvotes, negative comments, and reports. In this way, participation and algorithms come together to co-produce normative bodily standards. These can be seen on the default algorithm, which is consistently dominated by photographs of young, white, slender women. These practices produce body standards on Reddit Gonewild by valuing certain kinds of bodies and devaluing others. They also reproduce body standards. By having a homepage display homogenous bodies, this informs new participants of the kinds of content that is welcome in this particular subreddit. People whose bodies are similar to the ones featured, like the images circulated on IRC of 'young blonde bombshells [...] with inexhaustible drives' (Slater 1998, 97), are implicitly encouraged to participate in exhibiting photos on Gonewild, while people whose bodies do not conform may not even submit their photos. Each decision to submit (or not submit) a photograph, each upvote, each comment is a micro-interaction that represents a negotiation of the platform's power.

Furthermore, these micro-interactions are negotiated, because they occur within the dynamic rules of the site. There are several layers of regulation on Reddit, beginning with the user agreement and privacy policy, which are legal documents that outline the site's terms of use. Reddit-wide rules and Rediquette, the informal expression of Reddit's values, inform the subreddit-specific rules, which are displayed on the sidebar of each subreddit, and detailed in a separate Frequently Asked Questions document. These regulation layers reflect the 
difference in governance of the entire site, which is the responsibility of Reddit's owners, and individual subreddits, which are moderated by volunteers.

Rules on Reddit Gonewild change over time in response to the practices of Redditors, which can replicate or deviate from the Gonewild's norms (see Meese 2014). For example, when moderators became aware of people submitting photographs that were not of themselves, they became concerned these images were being taken out of context and submitted to the site without the consent of the person in the image. To address this, they introduced a verification system: posters had to include a handwritten sign with their Reddit username, the subreddit name, and the date, to prove the photo's originality and intent that it be included on Gonewild. When this verification system was exploited by some individuals, who would manipulate the photos to make the signs read something else, moderators amended the requirements so posters had to crumple the paper sign, then take a photo of the uncrumpled sign, to create angles in the paper, which is more difficult to manipulate with image editing software (xs51 2014). In this way, Redditors can subvert the rules and these negotiations with moderators, underlining how important the social established norms are in organising practice on the platform. However, the platform's power can be reasserted through the ongoing algorithmic sorting of content posted to the site, leading to ongoing tensions about which kinds of content makes it to the homepage.

\section{Conclusion: Social practice and norms}

The above examples reveal how practice plays a key role in entrenching particular social and behavioural norms on social media platforms, and outline how practices interact with emerging forms of algorithmic processing. The dynamic nature of practice compounds the already complex state of platform politics, and raises a series of interesting questions for 
wider discussions around regulation on social media platforms. Firstly, our paper has underlined the fact that social practice directly influences normative behaviours on Facebook, and co-produces the normative environment of Gonewild. Subsequently, we argue that social practice should be viewed as a foundational site of regulation ${ }^{1}$, one that operates in relation to platform affordances in order to shape and constitute the social media environment. However, practice can either reproduce, or deviate from, aspirational goals set by platform operators or other actors (such as government or advertisers).

Kathy Bowrey (1996) argues that individuals rarely engage with formal law, or 'law on the books'. Instead, we 'gain our understanding of the law informally; we grasp it in our day to day experience and interactions in the world'. We suggest that, in line with this position; we also 'grasp' forms of regulation through practice. Platforms have preferred processes for producing or distributing content, and ideals about the environment in which these engagements take place. However, as we all know, no one actually reads the end-user license agreement (EULA) (see de Zwart 2010), examines the Terms of Service (ToS) (Humphreys 2013), or shares everything on Facebook as Mark Zuckerberg would like us to do. Instead, norms are established on these platforms through a series of ongoing social engagements. While the platform is no doubt important, as our examples underline, it is often the actions of other individuals that go on to influence practices on each platform. Practice, therefore, becomes not a method from which regulation is reproduced, but instead a dynamic site from which norms (such as those around sharing or nudity) are instantiated, articulated, and negotiated with others.

Importantly, because these practices are socially developed, they can deviate from the wishes, and affordances, of a social media platform. In deviating, they can also go on to influence the use of that platform significantly. For example, the norm of 'oversharing' runs 
counter to the goals of Facebook, which encourages individuals to 'Share what's new in your life'. However, this norm currently shapes cultures of use on Facebook, and no amount of platform rhetoric or restructuring of platforms will alter this social norm around sharing over the next few years. Similarly, the recursive interactions between Reddit algorithms, the production of amateur pornography, and the voting system establish a normative bodily ideal. While Gonewild aspires to be a 'safe, mature, low-pressure environment for true exhibitionists' (xs51 2014), a combination of practice and algorithmic operations leads the subreddit to welcome some exhibitionists more than others.

Of course, this focus on practice does not wholly deny the importance of the algorithm or the platform in our current moment. What this paper does is underline the extent to which practice can also support and reinforce these technical affordances, structures, and processes. While the importance of algorithms has been noted in the literature, we outline how online interactions are equally important in shaping performances on social media platforms. While an open vulva may be considered 'controversial' by an algorithm, it is only through practice (upvoting or downvoting), and human intervention through programming, that algorithms are able to make these choices. Of course this is a recursive practice, but it underlines the necessity of not just examining algorithms, but also the contexts in which these algorithms are deployed through careful, longitudinal, qualitative research, such as the study we have outlined here.

These contrasting narratives of divergence and support from the aspirations of algorithms or platforms are not contradictory. What these narratives do is underline the dynamic nature of practice as well as its centrality in the current digital environment. It outlines an alternative trajectory of regulation, which is not based privately established 
processes (EULAs, ToS, or 'flags') but instead one grounded in sociality, reciprocity, and perhaps even the maintenance of a particular community ideal. By considering practice, this paper outlines how individuals shape these cultures of regulation, and contribute to the negotiation of existing regulatory frameworks and algorithmic processes. Future research could further explore ways in which negotiations between regulation and practice shape both the user experience and the affordances of social media platforms.

\section{Notes}

1. This claim refers to the work of Laura Murray, S. Tina Piper, and Kirsty Robinson (2014 et al.), who make a similar claim in relation to intellectual property law.

\section{Notes on contributors}

Jenny Kennedy is a Research Fellow in Computing and Information Systems at The University of Melbourne. She recently submitted a PhD in Media Studies at Swinburne University of Technology. Her primary research is on how digital technologies afford and complicate practices of sharing. Broadly, Jenny's research interests are media theories of everyday life, social discourses around technology use, and material culture.

James Meese is a Lecturer at the School of Communication, University of Technology, Sydney. His research interests include information law, mobile media, media regulation and sports media. His research has been published in Television and New Media, The European Journal of Cultural Studies and the Media Arts Law Review. 
Emily van der Nagel is a $\mathrm{PhD}$ candidate at Swinburne University of Technology in Melbourne, Australia. Her thesis investigates anonymity on social media. Her research has been published in Scan: Journal of Media Arts Culture and First Monday.

\section{Acknowledgements}

This article is an output from a Cultural Studies Association of Australasia Intermezzo Symposium held at Swinburne University of Technology. The authors wish to thank CSAA and Swinburne University of Technology for their financial support.

\section{References}

Barnes, Barry. 2001. "Practice as collective action." In The Practice Turn in Contemporary Theory, edited by Theodore R Schatzki, Karin Knorr-Cetina and Eike von Savigny, 17-28. Routledge: London.

Bourdieu, Pierre. 1984. Distinction: A social critique of the judgement of taste. Harvard University Press: Cambridge.

Bowrey, Kathy. 1996. “Who’s Writing Copyright's History?” European Intellectual Property Review 18: 322-329.

Bucher, Tania. 2012. "Want to Be on the Top? Algorithmic Power and the Threat of Invisibility on Facebook.” New Media \& Society 14 (7) 1164-1180.

Burgess, Jean. 2014. “From 'Broadcast yourself' to 'Follow your interests': Making over social media.” International Journal of Cultural Studies [published online before print].

Burke, Moira, Cameron Marlow, and Thomas Lento. "Social network activity and social well-being." Postgraduate Medical Journal 85: 455-459. 
Couldry, Nick. 2012. Media, Society, World: Social theory and digital media practice. Polity: London.

Crawford, Kate, and Tarleton Gillespie. 2014. "What is a flag for? Social media reporting tools and the vocabulary of complaint." New Media \& Society [published online before print].

De Zwart, Melissa. 2010. "Contractual communities: effective governance of virtual worlds." University of New South Wales Law Journal 33 (2): 605-627.

Flick, Uwe. 2009. An Introduction to Qualitative Research. Sage: London.

Gerlitz, Carolin, and Anne Helmond. 2013. "The like economy: Social buttons and the dataintensive web," New Media Society 15 (8): 1348-1365.

Gibbs, Martin, James Meese, Michael Arnold, Bjorn Nansen, and Marcus Carter. 2014, “\#Funeral and Instagram: death, social media, and platform vernacular.” Information, Communication \& Society 18 (3): 255-268.

Gillespie, Tarleton. 2013. “The Relevance of Algorithms.” In Media Technologies: Essays on Communication, Materiality, and Society, edited by Tarleton Gillespie, Pablo J. Boczkowski, and Kirsten A. Foot: 167-193. MIT Press: Cambridge.

Gonewild. 2014. Reddit. Accessed 11 December 2014. http://en.reddit.com/r/gonewild Holstein, James A. and Jaber F. Gubrium. 2002. Handbook of Interview Research: Context and Method, Sage: London.

Humphreys, Sal. 2013. "Predicting, Securing and Shaping the Future: Mechanisms of Governance in Online Social Environments.” International Journal of Media \& Cultural Politics 9 (3): 247-258. 
Kennedy, Jenny. 2013. "Rhetorics of Sharing: Data, Imagination and Desire.” In Unlike Us Reader: Social Media Monopolies and their Alternatives, edited by Geert Lovink and Miriam Rasch, 127-136. Institute of Network Cultures: Amsterdam.

Krippendorf, Klaus. 2013. Content Analysis: An Introduction to Its Methodology. 3rd ed. Sage: Los Angeles.

Meese, James. 2014. “'It belongs to the Internet': Animal images, attribution norms and the politics of amateur media production." M/C Journal (17): 2.

Merten, Don E. 1999. "Enculturation into secrecy among junior high school girls." Journal of Contemporary Ethnography 28 (2): 107-137.

Milner, Ryan M. 2013. “Pop Polyvocality: Internet Memes, Public Participation, and the Occupy Wall Street Movement.” International Journal of Communication 7: 23572390.

Murray, Laura J., S. Tina Piper, and Kirsty Robertson. 2014. Putting Intellectual Property in Its Place: Rights Discourses, Creative Labor, and the Everyday. Oxford University Press: New York.

Salihefendic, Amir. 2010. "How Reddit ranking algorithms work." Hacking and Gonzo, 23 November. Accessed 12 June 2013. http://amix.dk/blog/post/19588

Schneider, Todd W. 2014. "The Reddit Front Page is Not a Meritocracy." Todd W. Schneider, 6 November. Accessed 11 November 2014. http://toddwschneider.com/posts/the-reddit-front-page-is-not-a-meritocracy/

Shove, Elizabeth, and Mika Pantzar. 2005. "Consumers, Producers and Practices: Understanding the Invention and Reinvention of Nordic Walking." Journal of Consumer Culture 5 (1): 43-64. 
Silverman, David. 2011. “Data Analysis.” In Interpreting Qualitative Data. 4th ed: 57-86. Sage: London.

Slater, Don. 1998. "Trading sexpics on IRC: Embodiment and authenticity on the internet." Body \& Society 4 (4): 91-117.

van Dijck, José. 2013. "'You have one identity': Performing the self on Facebook and LinkedIn.” New Media and Society 35 (2): 199-215.

Wang, Yang, Gregory Norcie, Saranga Komanduri, Alessandro Acquisti, Pedro Giovanni Leon, and Lorrie Faith Cranor. 2011. "I regretted the minute I pressed share: A qualitative study of regrets on Facebook." Proceedings of the Seventh Symposium on Usable Privacy and Security: ACM.

Warde, Alan. 2005. "Consumption and theories of practice." Journal of Consumer Culture 5 (2): 131-153.

xs51. 2014. “The Gonewild FAQ". Reddit. Accessed 9 September 2014. http://www.reddit.com/r/gonewild/wiki/faq 